\title{
Taking More Gastroscopy Images Increases the Detection Rate of Clinically Significant Gastric Lesions: Validation of a Systematic Screening Protocol for the Stomach
}

\author{
Ja In Lee, Joon Sung Kim, Byung-Wook Kim, Cheal Wung Huh \\ Department of Internal Medicine, Incheon St. Mary's Hospital, College of Medicine, The Catholic University of Korea, Incheon, Korea
}

Background/Aims: For systematic screening protocol for the stomach (SSS), 22 gastroscopy images are considered sufficient to avoid blind spots during gastroscopy. The aim of this study was to investigate the relationship between the number of gastroscopy images taken during the gastroscopy procedure and the detection rate of clinically significant gastric lesions (CSGLs).

Materials and Methods: We retrospectively reviewed the data obtained from a cohort of consecutive subjects at a health promotion center. The primary outcome measure was the detection rate of CSGLs per endoscopist, according to the number of gastroscopy images. We also analyzed whether all the CSGLs were detected via SSS.

Results: The mean number of gastroscopy images obtained by eight endoscopists was 27.6 10.5 in 2,912 subjects without CSGLs and without biopsies. Among the 5,970 subjects who underwent gastroscopy by the eight endoscopists, 712 CSGLs were detected in 551 subjects. Fifty-six CSGLs (7.9\%) in 55 subjects (10.0\%) were not detected during the SSS. Photo-endoscopists who took more images achieved a higher detection rate of CSGLs than those who took fewer images (adjusted OR 2.07, 95\% CI 1.41 3.05; $P<0.0001)$.

Conclusions: The modified SSS, which included 22 SSS images, the fundus, and the saddle area, detected significantly more CSGLs. This modified SSS should be validated with further prospective studies. (Korean J Helicobacter Up Gastrointest Res 2020;20:225-232)

Key Words: Gastric epithelial neoplasia; Gastroscopy; Systematic screening protocol

\section{INTRODUCTION}

Taking multiple images during diagnostic esophagogastroduodenoscopy (EGD) is an important quality indicator and is recommended by endoscopy societies from various regions. European Society of Gastrointestinal Endoscopy (ESGE) recommended taking eight anatomical landmark images, including two images for the esophagus, four images for the stomach, and two images for the duodenum, with a focus on demonstrating the completeness of the EGD in 2001. ${ }^{1}$ In the updated version in 2016, ESGE recommended taking a total of 10 images, adding one each in the esophagus and in the stomach. ${ }^{2}$ Recently systematic screening protocol for the stomach (SSS) focused on the

Received: February 19, 2020 Revised: March 23, 2020 Accepted: March 30, 2020

Corresponding author: Byung-Wook Kim

Department of Internal Medicine, Incheon St. Mary's Hospital, College of Medicine, The Catholic University of Korea, 56 Dongsu-ro, Bupyeong-gu, Incheon 21431, Korea Tel: +82-32-280-5052, Fax: +82-32-280-5987, E-mail: gastro@catholic.ac.kr detection of early gastric cancer (EGC) has been suggested in Japan. ${ }^{3}$ This protocol proposed to take 22 gastroscopy images as a standardized protocol for mapping the entire stomach to avoid blind spots during EGD. The locations of 22 images of the SSS are as follows; in the antegrade view, endoscopic images of the four quadrants of the gastric antrum, lower body, and middle-upper body; in the retroflex view, endoscopic images of the four quadrants of the fundus and cardia, and three quadrants of middle-upper body and angle. ${ }^{3}$ However, to our knowledge, this protocol has not been validated for other gastric lesions.

Most of endoscopists might agree that taking enough endoscopy images during EGD is an important indicator of completeness for EGD. However, there has been no report on the relationship between the number of gastroscopy images and the detection rate of clinically significant gastric lesions (CSGLs) during EGD. The aim of this study was to investigate the relationship between the number of gastroscopy images and the detection

Copyright $\odot 2020$ Korean College of Helicobacter and Upper Gastrointestinal Research

(a) The Korean Journal of Helicobacter and Upper Gastrointestinal Research is an Open-Access Journal. All articles are distributed under the terms of the Creative Commons Attribution Non-Commercial License (http:// creativecommons.org/licenses/by-nc/4.0) which permits unrestricted non-commercial use, distribution, and reproduction in any medium, provided the original work is properly cited. 
rate of CSGLs. We also tried to analyze whether all the CSGLs might be included within SSS.

\section{MATERIALS AND METHODS}

\section{Study design and participants}

We retrospectively reviewed the medical records and digitally stored gastroscopy images of consecutive subjects who underwent screening EGD at a health promotion center in a tertiary referral hospital, between May 2017 and November 2017. We collected patients' data such as body mass index, alcohol, and cigarette smoking based on the questionnaire and physical examination charts. Subjects with gastrointestinal symptoms on the questionnaire were excluded.

Three board-certified endoscopists and five trainees were included in this study. Trainees performed at least 500 cases of EGD under supervision before participating in this study. All EGDs were performed using standard upper gastrointestinal endoscopes (GIF Q260 or GIF H260; Olympus, Tokyo, Japan). We excluded subjects with 1) $<18$ years old, 2) prominent gastric lesions such as gastric varices, polyposis, and advanced gastric cancer that were immediately detected at the time of EGD, 3) anatomical deformity due to gastrectomy or ulcer scarring, 4) incomplete study due to food stasis or other causes, 5) loss of digital images, and 6) inadequately blurred gastroscopy images. The Institutional Review Boards of Hospital approved this study (No: OC18RESI0119).

\section{Number of gastroscopy images}

Twenty-two gastroscopy images as in the SSS and images of other regions were counted and duplicated images at the same sites were discarded. Mean number of gastroscopy images for each endoscopist was calculated from cases without CSGLs and without biopsy. Endoscopists were classified into two groups based on their mean number of gastroscopy images using cut-off of 22 as in the SSS; one who took more endoscopic images (more photo-endoscopist) and the other who took less endoscopic images (less photo-endoscopist).

\section{Observation time of stomach and frequency of biopsy rates}

Observation time in the stomach was calculated from the images which showed the time that the image was taken. Observation time in the stomach was defined as the time interval between the initial image of stomach and the last image of stomach without biopsy. Using mean observation time of eight endoscopists as cut-off time, they were classified into fast endoscopists and slow endoscopists as in the previous study. ${ }^{4}$ The eight endocopists were also classified into two groups according to the mean frequency of biopsy rate.

\section{Outcomes and measurement}

\section{1) Primary outcome}

The primary outcome was the detection rate of CSGLS according to the number of endoscopic images taken. We defined CSGLs as EGC, adenoma/dysplasia, neuroendocrine tumor (NET), mucosa-associated lymphoid tissue (MALT) lymphoma, subepithelial tumor (SET), polyp, and benign gastric ulcer (open ulcer only) in this study. All the lesions at least $5 \mathrm{~mm}$ in diameter was calculated. Lesions of the esophagus and duodenum were not investigated in this study. Among the CSGLs, EGC, adenoma/dysplasia, NET, MALT lymphoma, and benign gastric ulcer were confirmed histologically while SET and polyp were diagnosed endoscopically. We classified CSGLs into neoplastic lesions (EGC, adenoma/dysplasia, NET, MALT lymphoma) and non-neoplastic lesions (SET, polyp, benign gastric ulcer). The relationship between the number of gastroscopy images and the detection rate CSGLs were analyzed.

\section{2) Secondary outcome}

The secondary outcome was to validate whether all the CSGLs were included in the SSS. The ratio of CSGLS out of SSS over the total number of CSGLs were calculated. Two board-certified endoscopists reviewed and confirmed the locations of CSGLs. Stomach was divided into seven parts longitudinally and into four quad- 
rants circumferentially to analyze the location of lesion. Seven parts were designated as antrum, low body, mid body, high body, angle, cardia, and fundus while four quadrants were designated as anterior wall, posterior wall, lesser curvature, and greater curvature., ${ }^{5,6}$ We defined the boundary between antrum and low body in the greater curvature as the area where gastric fold ends. We also defined the boundary between high body and fundus in the greater curvature as saddle area.

\section{Statistical methods}

Student's t-test was used to analyze the differences in detection rate of CSGLs between more photo-endoscopists and less photo-endoscopists. Chi-squared test or Fisher's exact test were used to compare categorical variables. Descriptive data was presented as mean $\pm \mathrm{SD}$. The relationship between mean number of gastroscopy images and detection rate of CSGLs for each endoscopist was analyzed using Pearson's correlation coefficient. Logistic regression analysis was used to identify independent factors affecting the detection rate of CSGLs. Variables that showed a significant association with CSGLS in the univariate analysis were included in the multivariate analysis. $P$-values less than 0.05 were considered statistically significant. Statistical analyses were performed using SPSS version 20.0 (IBM Corp, Armonk, NY, USA).

\section{RESULTS}

\section{Subjects}

A total of 6,061 subjects were reviewed and a total of 5,970 subjects were analyzed in this study. Excluded subjects were as follows; gastric varix $(n=1)$, polyposis $(n=1)$, advanced gastric cancer $(n=1)$, previous gastrectomy $(n=36)$, incomplete study due to food stasis $(n=14)$, other causes $(n=18)$, and loss of digital images $(n=20)$. Table 1 shows the characteristics of the subjects and the CSGLs detected in this study. The mean age of subjects was $56.8 \pm 10.3$ years, and $2,852(47.8 \%)$ were males.
Table 1. Baseline Characteristics of the Subjects and Observed Clinically Significant Gastric Lesions

\begin{tabular}{lc}
\hline Subjects & Total $(\mathrm{n}=5,970)$ \\
\hline Age (years) & $56.8 \pm 10.3$ \\
Male & $2,852(47.8)$ \\
Body mass index $\left(\mathrm{kg} / \mathrm{m}^{2}\right)$ & $24.2 \pm 3.0$ \\
Smoking & $547(9.2)$ \\
Alcohol & $1,732(29.0)$ \\
Clinically significant gastric lesions & 712 lesions/551 subjects \\
Early gastric cancer & $7 / 7$ \\
Adenoma/dysplasia & $13 / 13$ \\
Neuroendocrine tumor & $1 / 1$ \\
MALT lymphoma & $1 / 1$ \\
Subepithelial tumor & $166 / 152$ \\
Benign gastric ulcer & $99 / 57$ \\
Polyp & $425 / 320$ \\
\hline
\end{tabular}

Values are presented as mean \pm standard deviation or $\mathrm{n}(\%)$ unless otherwise indicated.

MALT, mucosa-associated lymphoid tissue.

\section{Number of gastroscopy images and detection rate of CSGLS}

Among 5,970 subjects, there was no CSGL and biopsy was not performed in 2,912 subjects. Mean number of endoscopy images and observation time for each endoscopist were calculated from these 2,912 subjects.

Mean number of gastroscopy images for more photo-endoscopists was $32.6 \pm 8.0$ and that of less photo-endoscopists was $15.9 \pm 4.5(P=0.001)$. The detection rates of CSGLs for each endoscopist were from $1.89 \%$ to $17.12 \%$ (Table 2). Mean number of gastroscopy images and the detection rate of CSGLS were positively correlated (Fig. 1).

Using 22 images of the SSS as cut-off, six endoscopists were classified as more photo-endoscopists and two endoscopists were classified as less photo-endoscopists. More photo-endoscopists had a higher detection rate of CSGLs than less photo-endoscopists (10.36\% vs. 3.91\%, $P<0.0001$, in terms of subjects). However, there was no difference in detection rate of EGC in two groups ( $0.10 \%$ vs. $0.19 \%$, $P=0.355$, in terms of subjects). Also there was no difference in the detection rates of dysplasia/adenoma ( $0.24 \%$ vs. $0.09 \%, P=0.712$, in terms of the subjects), NET (0.02\% vs. 
Table 2. Characteristics of Each Endoscopist and Detection Rate of Clinically Significant Gastric Lesions

\begin{tabular}{|c|c|c|c|c|c|}
\hline \multirow[b]{2}{*}{ Endoscopist } & \multirow{2}{*}{$\begin{array}{c}\text { Mean number of } \\
\text { gastroscopic images }^{\mathrm{a}}\end{array}$} & \multirow{2}{*}{$\begin{array}{l}\text { Mean examination } \\
\text { time (seconds) }\end{array}$} & \multirow[b]{2}{*}{ Biopsy rate $(\%)^{b}$} & \multicolumn{2}{|c|}{ Detection rate } \\
\hline & & & & $\begin{array}{l}\text { Number of } \\
\text { EGDs }\end{array}$ & $\begin{array}{c}\text { Number of subjects } \\
\text { with CSGLs }\end{array}$ \\
\hline A & $29.1 \pm 4.6$ & $135.5 \pm 43.4$ & $61.0(459 / 752)$ & 752 & $74(9.84)$ \\
\hline B & $26.9 \pm 5.3$ & $94.3 \pm 29.0$ & $23.9(248 / 1,036)$ & 1,036 & $83(8.01)$ \\
\hline $\mathrm{C}$ & $42.1 \pm 5.0$ & $126.6 \pm 40.1$ & $75.2(610 / 811)$ & 811 & $139(17.12)$ \\
\hline $\mathrm{D}$ & $31.0 \pm 8.7$ & $86.6 \pm 43.7$ & $41.7(305 / 731)$ & 731 & $58(7.93)$ \\
\hline $\mathrm{E}$ & $38.3 \pm 4.3$ & $113.9 \pm 39.2$ & $40.6(378 / 930)$ & 930 & $94(10.11)$ \\
\hline $\mathrm{F}$ & $14.3 \pm 4.4$ & $45.8 \pm 23.9$ & $21.8(102 / 468)$ & 468 & $30(6.41)$ \\
\hline G & $17.0 \pm 4.2$ & $62.1 \pm 28.1$ & $9.5(56 / 592)$ & 592 & $11(1.89)$ \\
\hline $\mathrm{H}$ & $37.6 \pm 6.0$ & $74.6 \pm 20.1$ & $37.2(242 / 650)$ & 650 & $62(9.54)$ \\
\hline Total & $27.6 \pm 10.5$ & $87.9 \pm 42.7$ & $40.2(2,400 / 5,970)$ & 5,970 & $551(9.23)$ \\
\hline Correlation coefficient ( $P$-value) & & $0.70(0.055)$ & $0.75(0.032)$ & $0.55(0.156)$ & $0.82(0.012)$ \\
\hline
\end{tabular}

Values are presented as mean \pm standard deviation or $\mathrm{n}(\%)$ unless otherwise indicated.

EGD, esophagogastroduodenoscopy; CSGL, clinically significant gastric lesion.

aAnalyzed from 2,912 subjects without CSGLs and without biopsy.

${ }^{\mathrm{b}}$ Analyzed from total 5,970 subjects.

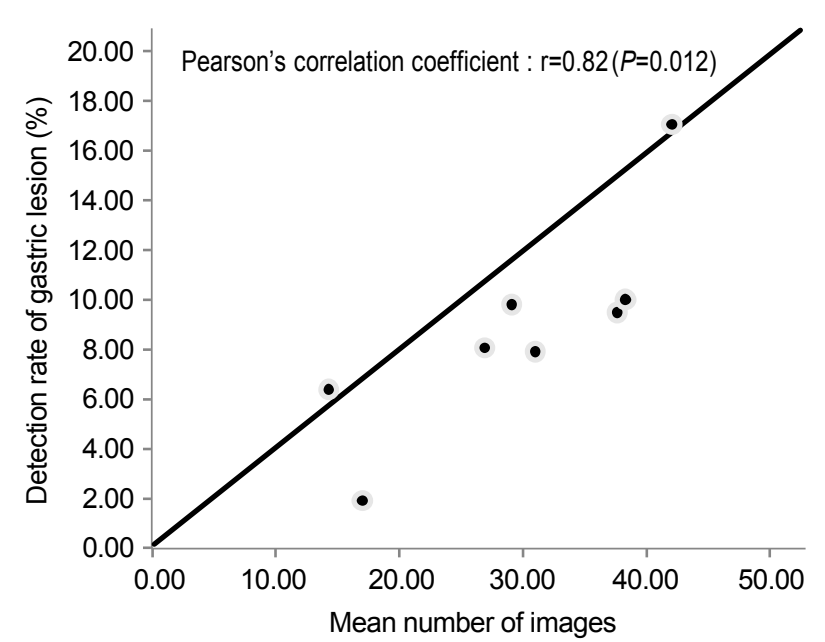

Fig. 1. The detection rate of the gastric lesion according to the mean number of images obtained during gastroscopy by eight endoscopists.

$0.00 \%, P=1.00$, in terms of the subjects), and MALT lymphoma ( $0.02 \%$ vs. $0.00 \%, P=1.00$, in terms of the subjects) between the two groups.

\section{Number of gastroscopy images, observation time, and frequency of biopsy}

Mean observation time measured in 2,912 subjects without CSGLs and without biopsy by the eight endoscopists was $87.9 \pm 42.7$ seconds. With Pearson's correlation test, there was a tendency of correlation between mean number of images and observation time for each endoscopists, although statistically not significant $(\mathrm{r}=0.70 ; P=0.055)$.

We classified the endoscopists into fast endoscopists $(n=4)$ and slow endoscopists ( $n=4)$ using mean observation time for eight endoscopists as cut-off of 87 seconds. Slow endoscopists showed a significantly higher detection rate of CSGLs than fast endoscopists (11.1\% vs. 6.6\%, $P<0.0001)$.

The eight endoscopists were also divided into high biopsy rate endoscopists $(\mathrm{n}=4)$ and low biopsy rate endoscopists ( $\mathrm{n}=4$ ) using mean biopsy rate for eight endoscopists as cut-off of $40.2 \%$. High biopsy rate endoscopists showed a higher detection rate of CSGLs than low biopsy rate endoscopists $(11.3 \%$ vs. $6.8 \%, P<0.0001)$.

\section{Factors associated with detection rate of CSGLS}

Table 3 shows logistic regression analysis to identify independent factors affecting the detection rate of CSGLs. In univariate analysis, old age ( $\geq 65$ years), male, longer observation time, and more photo-endoscopists were associated with detection rate of CSGLs. However, there was no significant difference in detection rate of CSGLs by endoscopist experience (OR 0.91, 95\% CI 0.76 1.11; $P=0.364$ ). 
Table 3. Factors Associated with the Detection Rate of Clinically Significant Gastric Lesions

\begin{tabular}{|c|c|c|c|c|c|c|}
\hline \multirow{2}{*}{ Factors } & \multirow{2}{*}{$\begin{array}{l}\text { Total subjects } \\
\quad(n=5,790)\end{array}$} & \multirow{2}{*}{$\begin{array}{c}\text { Subjects with } \\
\text { CSGLs }(\mathrm{n}=551)\end{array}$} & \multicolumn{2}{|c|}{ Univariate analysis } & \multicolumn{2}{|c|}{ Multivariate analysis } \\
\hline & & & OR $(95 \% \mathrm{CI})$ & $P$-value & Adjusted OR (95\% CI) & $P$-value \\
\hline \multicolumn{7}{|l|}{ Age (years) } \\
\hline $18 \sim 64$ & $4,575(76.63)$ & $379(8.28)$ & Reference & & & \\
\hline$\geq 65$ & $1,395(23.37)$ & $172(12.33)$ & $1.56(1.29-1.89)$ & $<0.0001$ & $1.45(1.19-1.76)$ & $<0.0001$ \\
\hline \multicolumn{7}{|l|}{ Sex } \\
\hline Male & $2,852(47.77)$ & 339 (11.89) & $1.52(1.27-1.82)$ & $<0.0001$ & $1.40(1.16-1.69)$ & $<0.001$ \\
\hline Female & $3,118(52.23)$ & $212(6.80)$ & Reference & & & \\
\hline \multicolumn{7}{|l|}{ Smoking } \\
\hline Yes & $547(9.16)$ & $36(6.58)$ & $0.73(0.50-1.07)$ & 0.104 & & \\
\hline No & $1,847(30.94)$ & $162(8.77)$ & Reference & & & \\
\hline Unknown & $3,576(59.90)$ & $353(9.87)$ & $1.14(0.94-1.38)$ & 0.190 & & \\
\hline \multicolumn{7}{|l|}{ Alcohol } \\
\hline Yes & $1,732(29.01)$ & $128(7.39)$ & $0.72(0.56-0.93)$ & 0.013 & $0.85(0.65-1.11)$ & 0.228 \\
\hline No & $1,285(21.52)$ & $128(9.96)$ & Reference & & & \\
\hline Unknown & $2,953(49.46)$ & 295 (9.99) & $1.003(0.81-1.25)$ & 0.977 & $1.03(0.83-1.29)$ & 0.786 \\
\hline \multicolumn{7}{|c|}{ Examination time (seconds) } \\
\hline$<87$ & $2,441(40.89)$ & $161(6.60)$ & Reference & & & \\
\hline$\geq 87$ & $3,529(59.11)$ & $390(11.05)$ & $1.76(1.45-2.13)$ & $<0.001$ & $1.18(0.94-1.47)$ & 0.144 \\
\hline \multicolumn{7}{|c|}{ Number of images } \\
\hline$<22$ & $1,048(17.55)$ & $41(3.91)$ & Reference & & & \\
\hline$\geq 22$ & $4,922(82.45)$ & $510(10.36)$ & $2.84(2.05-3.93)$ & $<0.001$ & $2.07(1.41-3.05)$ & $<0.0001$ \\
\hline \multicolumn{7}{|l|}{ Experience } \\
\hline Board-certified & $1,711(28.66)$ & $105(6.14)$ & $0.91(0.76-1.11)$ & 0.364 & & \\
\hline Trainee & $4,259(71.34)$ & 447 (10.49) & Reference & & & \\
\hline
\end{tabular}

Values are presented as $\mathrm{n}(\%)$ unless otherwise indicated.

CSGL, clinically significant gastric lesions; OR, odds ratio; CI, confidence interval.

Table 4. Characteristics of Clinically Significant Gastric Lesions Not Included in Systematic Screening Protocol for the Stomach

\begin{tabular}{lccc}
\hline Diagnosis & Location & Number of lesions & Mean size of the lesions $(\mathrm{mm})$ \\
\hline Subepithelial tumor & Fundus & 23 & $5.6 \pm 2.8$ \\
& Saddle area & 3 & $7.0 \pm 1.7$ \\
Polyp & Fundus & 20 & $3.0 \pm 1.6$ \\
& Saddle area & 10 & $2.8 \pm 0.6$ \\
\hline
\end{tabular}

Values are presented as mean \pm standard deviation or $\mathrm{n}$.

In multivariate analysis, more photo-endoscopists detected CSGLs more commonly than less photo-endoscopists (adjusted OR 2.07, 95\% CI 1.41 3.05; $P<0.0001$ ). However, observation time did not affect the detection rate of CSGLs (adjusted OR 1.18, 95\% CI 0.94 1.47; $P=0.144$ ).

\section{Analysis on location of gastric lesions}

Among 712 CSGLs in 551 subjects, 56 (10.1\%) CSGLs in 50 subjects were located out of SSS. Of these 56 CSGLs located out of SSS, SETs were 26 (4.7\%) and polyps were 30 (5.4\%). Characteristics of CSGLs out of SSS are described in Table 4. Additional lesions were located in the fundus dome and in the saddle area. Examples 

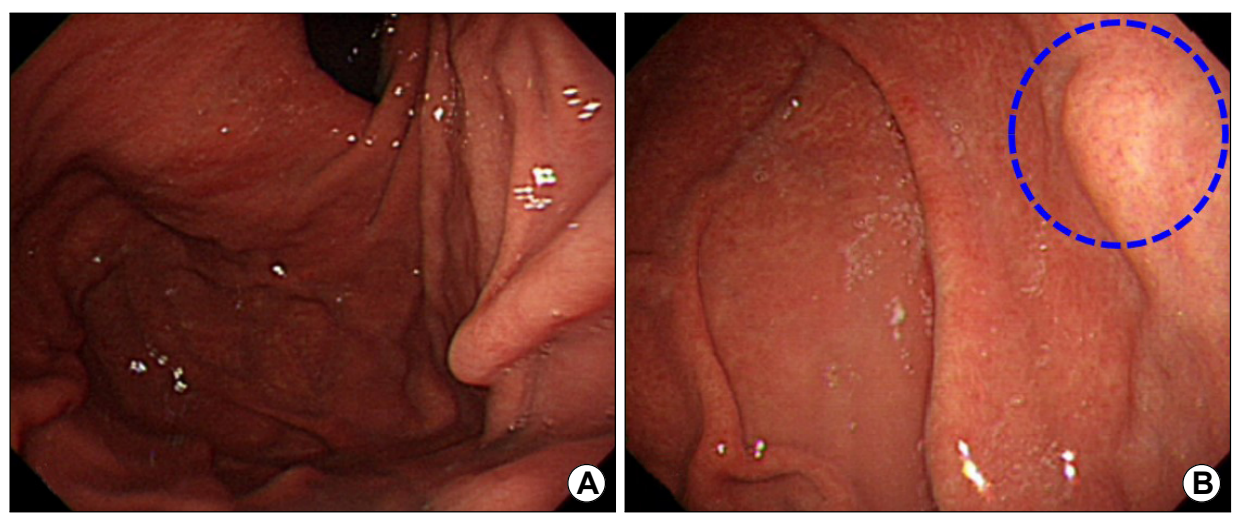

Fig. 2. Representative image of a subepithelial tumor (SET) not included in the systematic screening protocol for the stomach (SSS) at the gastric fundus. (A) In the retroflexed view, the SET is not observed in one of the SSS images. (B) In the antegrade view, the SET is detected at the boundary between the greater curvature side and the anterior wall side of the fundus, located left of saddle area (circle). The boundary is not included in the 22 images generated during the SSS.

are presented in Fig. 2.

\section{DISCUSSION}

While adenoma detection rate is known as the most ideal quality indicator in colonoscopy, ${ }^{7,8}$ there is no good quality indicator in EGD due to low incidence of EGC. Quality control in EGD consists of three categories: structure, procedure, and outcome, among which procedure component assesses endoscopists' performance during EGD and is associated with clinical outcome such as detection of tumor. ${ }^{9}$ Systematic image documentation to map the entire stomach is also needed by other several reasons. EGD is a subjective examination, and images from EGD can be interpreted very differently. ${ }^{10,11}$ Therefore, EGD must be objectified, and this can be possible by mapping the entire stomach. The mapping also helps establish a baseline endoscopic landscape for potential future endoscopic evaluation such as background mucosa, and can be ultimately cost-effective by avoiding repetitive EGD during a second opinion consultative process. ${ }^{12}$ Patients now have growing understanding on medical information and want to check their test results through images. In current situation where disputes in medical treatments are increasing, the mapping can be an effective tool to protect both patients and doctors.

In this study, we found that more photo-endoscopists showed a higher detection rate of CSGLs than less photo-endoscopists. Mean number of gastroscopy images and the detection rate of CSGLs were positively correlated. It seems likely that there was a tendency of somewhat higher detection rate of EGCs in more photo-endoscopists. However, it should be remembered that EGCs were found only in seven cases and sub-group analysis was not possible. So endoscopists should be vigilant to take more endoscopy images during diagnostic EGD.

As shown in Table 2, more photo-endoscopists tend to observe longer and to take biopsy more often than less photo-endoscopists. Observation time and biopsy rate were quite variable among the endoscopists. It is plausible that enthusiasm and interest of endoscopists are closely related to taking more pictures, more observation time, and more biopsy rate, which might result in more detection rate of CSGLs.

Our study showed that observation time did not affect the detection rate of CSGLs. However, there were some reports on the detection rate of gastric lesions and examination times. One previous study suggested that 7-minute examination time from insertion to removal of scope might identify a great number of high-risk gastric lesions. ${ }^{13}$ However, the examination time of this study included the esophagus, stomach, and duodenum, and we cannot conclude how long the endoscopists examined the stomach. In another study, withdrawal time of 3 minutes from the duodenum might increase the detection rate of upper gastrointestinal neoplasm. ${ }^{4}$ However, the purpose of EGD is not always focused on upper gastrointestinal neoplasm. Endoscopists should try to find every CSGLs. In our study, mean observation time for the stomach was 87.9 \pm 42.7 seconds, which is somewhat shorter compared to previous studies. There is no current consensus on observation time of the stomach during EGD. Thus, comparison of obtaining 
enough gastroscopy images in good quality versus examination time for the stomach in detection rate of CSGLs should be elucidated with further prospective studies.

We tried to validate the SSS in this study and found that about $10.6 \%$ of CSGLS were out of SSS. These CSGLS were mainly SETs or polyps and located in the fundus dome or saddle area. Cardia, posterior wall side of the body, and pylorus are known as blind spots in the stomach. ${ }^{14,15}$ However, SSS includes all of these sites while fundus dome and saddle area are blind spots. Although neoplastic lesions were not located in the fundus dome and saddle area in this study, neoplastic lesions can be found in this area. We would like to suggest a modified SSS as follows; 24 images including 22 SSS images and additional images for the fundus dome and saddle area.

Strength of this study is that we analyzed in a large scale including over 5,000 subjects without gastrointestinal symptoms. Despite this strength, there are several limitations in this study. First, this study has a retrospective design. However, we included all consecutive cases in a cohort with no missing data to reduce selection bias. Second, the number of endocsopists in the study were relatively small. Third, among the endoscopists who participated in this study, trainees with less experience were included. However, we analyzed the data after trainees performed 500 or more EGD under supervision. Since American Society of Gastrointestinal Endoscopy and European Diploma of Gastroenterology require 130 and 300 procedures required for competence, it is reasonable that trainees participated in this study have adequate knowledge and technique to detect EGC. ${ }^{16,17}$ Fourth, we did not identify the prior EGD and surveillance interval. Fifth, we analyzed the detection rate of CSGLs, not neoplastic lesions. However, one study showed that the detection rate of SET had positive correlation with the detection rate of EGC. ${ }^{18}$

In conclusion, mean number of gastroscopy images and the detection rate of CSGLs were positively correlated. Modified SSS including 22 SSS images and additional images for the fundus and saddle area might detect most of CSGLs. This modified SSS might be validated with further prospective studies.

\section{ACKNOWLEDGEMENT}

The authors thank Dr. Hyun Woo Lim who works in Department of Preventive Medicine, The Catholic University of Korea for his help in statistical consultation.

\section{CONFLICT OF INTEREST}

No potential conflict of interest relevant to this article was reported.

\section{ORCID}

Ja In Lee (1) https://orcid.org/0000-0002-8055-7903
Joon Sung Kim (1) https://orcid.org/0000-0001-9158-1012
Byung-Wook Kim (1) https://orcid.org/0000-0002-2290-4954
Cheal Wung Huh (1) https://orcid.org/0000-0001-7327-8503

\section{REFERENCES}

1. Rey JF, Lambert R; ESGE Quality Assurance Committee. ESGE recommendations for quality control in gastrointestinal endoscopy: guidelines for image documentation in upper and lower GI endoscopy. Endoscopy 2001;33:901-903.

2. Bisschops R, Areia M, Coron E, et al. Performance measures for upper gastrointestinal endoscopy: a European Society of Gastrointestinal Endoscopy (ESGE) quality improvement initiative. Endoscopy 2016;48:843-864.

3. Yao K. The endoscopic diagnosis of early gastric cancer. Ann Gastroenterol 2013;26:11-22.

4. Park JM, Huo SM, Lee HH, Lee BI, Song HJ, Choi MG. Longer observation time increases proportion of neoplasms detected by esophagogastroduodenoscopy. Gastroenterology 2017;153: 460-469.e1.

5. Burdan F, Rozylo-Kalinowska I, Szumilo J, et al. Anatomical classification of the shape and topography of the stomach. Surg Radiol Anat 2012;34:171-178.

6. Lee SH, Park YK, Cho SM, Kang JK, Lee DJ. Technical skills and training of upper gastrointestinal endoscopy for new beginners. World J Gastroenterol 2015;21:759-785.

7. Rex DK, Schoenfeld PS, Cohen J, et al. Quality indicators for colonoscopy. Gastrointest Endosc 2015;81:31-53.

8. Ruiz-Rebollo ML, Alcaide-Suárez N, Burgueño-Gómez B, et al. Adenoma detection rate and cecal intubation rate: quality indicators for colonoscopy. Gastroenterol Hepatol 2019;42: 253-255.

9. Park WG, Shaheen NJ, Cohen J, et al. Quality indicators for EGD. Gastrointest Endosc 2015;81:17-30.

10. Asfeldt AM, Straume B, Paulssen EJ. Impact of observer varia- 
bility on the usefulness of endoscopic images for the documentation of upper gastrointestinal endoscopy. Scand J Gastroenterol 2007;42:1106-1112.

11. Bendtsen F, Skovgaard LT, Sørensen TI, Matzen P. Agreement among multiple observers on endoscopic diagnosis of esophageal varices before bleeding. Hepatology 1990;11:341-347.

12. Tang SJ, Raju G. Endoscopic photography and image documentation. Gastrointest Endosc 2015;82:925-931.

13. Teh JL, Tan JR, Lau LJ, et al. Longer examination time improves detection of gastric cancer during diagnostic upper gastrointestinal endoscopy. Clin Gastroenterol Hepatol 2015;13: 480-487.e2.

14. Moon HS. Improving the endoscopic detection rate in patients with early gastric cancer. Clin Endosc 2015;48:291-296.

15. Ren W, Yu J, Zhang ZM, Song YK, Li YH, Wang L. Missed diagnosis of early gastric cancer or high-grade intraepithelial neoplasia. World J Gastroenterol 2013;19:2092-2096.

16. Beattie AD, Greff M, Lamy V, Mallinson CN. The European Diploma of Gastroenterology: progress towards harmonization of standards. Eur J Gastroenterol Hepatol 1996;8:403-406.

17. Eisen GM, Baron TH, Dominitz JA, et al. Methods of granting hospital privileges to perform gastrointestinal endoscopy. Gastrointest Endosc 2002;55:780-783.

18. Park $\mathrm{CH}$, Kim B, Chung H, et al. Endoscopic quality indicators for esophagogastroduodenoscopy in gastric cancer screening. Dig Dis Sci 2015;60:38-46. 